\title{
COMBINATORIAL PRINCIPLES FROM ADDING COHEN REALS
}

\author{
I. JUHÁSZ, L. SOUKUP, AND Z. SZENTMIKLÓSSY
}

\begin{abstract}
We first formulate several "combinatorial principles" concerning $\kappa \times \omega$ matrices of subsets of $\omega$ and prove that they are valid in the generic extension obtained by adding any number of Cohen reals to any ground model $V$, provided that the parameter $\kappa$ is an $\omega$-inaccessible regular cardinal in $V$.

Then in section 1 we present a large number of applications of these principles, mainly to topology. Some of these consequences had been established earlier in generic extensions obtained by adding $\omega_{2}$ Cohen reals to ground models satisfying $\mathrm{CH}$, mostly for the case $\kappa=\omega_{2}$.
\end{abstract}

\section{INTRODUCTION}

The last 25 years have seen a furious activity in proving results that are independent of the usual axioms of set theory, that is ZFC. As the methods of these independence proofs (e.g. forcing or the fine structure theory of the constructible universe) are often rather sophisticated, while the results themselves are usually of interest to "ordinary" mathematicians (e.g. topologists or analysts), it has been natural to try to isolate a relatively small number of principles, i.e. independent statements that a) are simple to formulate and b) are useful in the sense that they have many interesting consequences. Most of these statements, we think by necessity, are of combinatorial nature, hence they have been called combinatorial principles.

In this paper we propose to present several new combinatorial principles that are all statements about $\mathcal{P}(\omega)$, the power set of the natural numbers. In fact, they all concern matrices of the form $\langle A(\alpha, n):\langle\alpha, n\rangle \in \kappa \times \omega\rangle$, where $A(\alpha, n) \subset \omega$ for each $\langle\alpha, n\rangle \in \kappa \times \omega$, and, in the interesting cases, $\kappa$ is a regular cardinal with $c=2^{\omega} \geq \kappa>\omega_{1}$.

1991 Mathematics Subject Classification. 03E35,54A25.

Key words and phrases. Cohen forcing, combinatorial principles, almost disjoint family, thin tall Boolean algebra, tower, irreducible base, separable, countably tight.

The second author was supported by DFG (grant Ko 490/7-1).

The preparation of this paper was supported by the Hungarian National Foundation for Scientific Research grant no. 16391. 
We show that these statements are valid in the generic extensions obtained by adding any number of Cohen reals to any ground model $V$, assuming that the parameter $\kappa$ is a regular and $\omega$-inaccessible cardinal in $V$ ( i.e. $\lambda<\kappa$ implies $\left.\lambda^{\omega}<\kappa\right)$.

Then we present a large number of consequences of these principles, some of them combinatorial but most of them topological, mainly concerning separable and/or countably tight topological spaces. (This, of course, is not surprising because these are objects whose structure depends basically on $\mathcal{P}(\omega)$.)

The above formulated criteria a) and b) as to what constitutes a combinatorial principle are often contrary to each other: for more usefulness one often has to sacrifice some simplicity. It is not clear whether an ideal balance exists between them. It is up to the reader to judge if we have come close to this balance.

\section{The COMBINATORIAL PRINCIPLES}

The principles we formulate here are all statements on $\kappa \times \omega$ matrices of subsets of $\omega$ claiming - roughly speaking - that all these matrices contain large "submatrices" satisfying certain homogeneity properties.

To simplify the formulation of our results we introduce the following pieces of notation. If $S$ is an arbitrary set and $k$ is a natural number then let

$$
(S)^{k}=\left\{s \in S^{k}:|\operatorname{ran} s|=k\right\}
$$

and

$$
(S)^{<\omega}=\bigcup_{k<\omega}(S)^{k} .
$$

For $D_{0}, \ldots, D_{k-1} \subset S$ we let

$$
\left(D_{0}, \ldots, D_{k-1}\right)=\left\{s \in(S)^{k}: \forall i \in k\left(s(i) \in D_{i}\right)\right\} \text {. }
$$

Definition 2.1. If $S$ is a set of ordinals denote by $\mathcal{M}(S)$ the family of all $S \times \omega$-matrices of subsets of $\omega$, that is, $\mathcal{A} \in \mathcal{M}(S)$ if and only if $\mathcal{A}=\langle A(\alpha, i): \alpha \in S, i<\omega\rangle$, where $A(\alpha, i) \subset \omega$ for each $\alpha \in S$ and $i<\omega$. If $\mathcal{A}=\langle A(\alpha, i): \alpha \in S, i<\omega\rangle \in \mathcal{M}(S)$ and $R \subset S$ we define the restriction of $\mathcal{A}$ to $R, \mathcal{A}\lceil R$ in the straightforward way: $\mathcal{A}\lceil R=$ $\langle A(\alpha, i): \alpha \in R, i<\omega\rangle$. If $\mathcal{A}=\langle A(\alpha, i): \alpha \in S, i<\omega\rangle \in \mathcal{M}(S), t \in$ $\omega^{<\omega}$ and $s \in(S)^{|t|}$ then we let

$$
A(s, t)=\bigcap_{i<|t|} A(s(i), t(i)) .
$$

Now we formulate our first and probably most important principle that we call $C^{s}(\kappa)$. We also specify a weaker version of $C^{s}(\kappa)$ denoted 
by $C(\kappa)$ because in most of the applications (4.1, 4.3, 4.11, 4.14, 4.18, 4.24 ) we don't need the full power of $C^{s}(\kappa)$.

Definition 2.2. For $\kappa=\operatorname{cf}(\kappa)>\omega$ principle $C^{s}(\kappa)(C(\kappa))$ is the following statement:

For every $T \subset \omega^{<\omega}$ and $\mathcal{A} \in \mathcal{M}(\kappa)$ we have (1) or (2) below:

(1) there is a stationary (cofinal) set $S \subset \kappa$ such that if $t \in T$ and $s \in(S)^{|t|}$ then

$$
A(s, t) \neq \emptyset
$$

(2) there are $t \in T$ and stationary (cofinal) subsets $D_{0}, D_{1}, \ldots, D_{|t|-1}$ of $\kappa$ such that for every $s \in\left(D_{0}, \ldots, D_{|t|-1}\right)$ we have

$$
A(s, t)=\emptyset \text {. }
$$

Next we formulate a dual version of principles $C^{s}(\kappa)$ and $C(\kappa)$. Although we don't yet know any application of principles $\hat{C}^{s}(\kappa)$ and $\hat{C}(\kappa)$, for the sake of completeness we include their definitions here. Let us remark that we don't know whether $C^{s}(\kappa)(C(\kappa))$ implies $\hat{C}^{s}(\kappa)(\hat{C}(\kappa))$ or vice versa.

Definition 2.3. If $\kappa=\operatorname{cf}(\kappa)>\omega$, then principle $\hat{C}^{s}(\kappa)(\hat{C}(\kappa))$ is the following statement:

For every $T \subset \omega^{<\omega}$ and $\mathcal{A} \in \mathcal{M}(\kappa)$ we have (1) or (2) below:

(1) there is a stationary (cofinal) set $S \subset \kappa$ such that for each $t \in T$ and $s \in(S)^{|t|}$

$$
|A(s, t)|<\omega
$$

(2) there are $t \in T$ and stationary (cofinal) subsets $D_{0}, D_{1}, \ldots, D_{|t|-1}$ of $\kappa$ such that for every $s \in\left(D_{0}, \ldots, D_{|t|-1}\right)$ we have

$$
|A(s, t)|=\omega .
$$

Let us remark that in the "plain" dual of principle $C^{s}(\kappa)$ we should have $|A(s, t)|=\emptyset$ in 2.3(1) and $|A(s, t)| \neq \emptyset$ in 2.3(2), but this "principle" is easily provable in ZFC.

The principles $D(\kappa)$ and $D^{s}(\kappa)$ that we introduce next easily follow from $C(\kappa)$ and $C^{s}(\kappa)$, respectively, but as their formulation is much simpler, we thought it to be worth while to have them as separate principles. We first give two auxiliary definitions.

Definition 2.4. If $\mathcal{A}=\langle A(\alpha, i): \alpha<\kappa, i<\omega\rangle \in \mathcal{M}(\kappa)$, then we set

$$
\hat{\mathcal{A}}=\{Y \subset \omega:|\{\alpha<\kappa: \exists i<\omega A(\alpha, i) \subset Y\}|=\kappa\}
$$

and

$$
\hat{\mathcal{A}}^{s}=\{Y \subset \omega:\{\alpha<\kappa: \exists i<\omega A(\alpha, i) \subset Y\} \text { is stationary in } \kappa\} .
$$


Definition 2.5. A matrix $\mathcal{A}=\langle A(\alpha, i): \alpha \in S, i \in \omega\rangle$ is called $\omega$-adic if for each $t \in \omega^{<\omega}$ and $s \in(S)^{|t|}$ we have $A(s, t) \neq \emptyset$.

Now we can formulate $D^{s}(\kappa)(D(\kappa))$ as follows.

Definition 2.6. For $\kappa=\operatorname{cf}(\kappa)>\omega$ principle $D^{s}(\kappa)(D(\kappa))$ is the following statement:

If $\mathcal{A} \in \mathcal{M}(\kappa)$ and $\hat{\mathcal{A}}^{s}(\hat{\mathcal{A}})$ is centered then there is a stationary (cofinal) set $S \subset \kappa$ such that $\mathcal{A}\lceil S$ is $\omega$-adic.

Theorem 2.7. $C^{s}(\kappa)(C(\kappa))$ implies $D^{s}(\kappa)(D(\kappa))$.

Proof. We give the proof only for $D^{s}(\kappa)$ because the same argument works for $D(\kappa)$.

Let $\mathcal{A} \in \mathcal{M}(\kappa)$ and put $T=\omega^{<\omega}$. By $C^{s}(\kappa)$ either 2.2(1) or 2.2(2) holds.

If $S \subset \kappa$ witnesses $2.2(1)$ for our $T$ then $\mathcal{A}\lceil S$ is clearly $\omega$-adic. So it is enough to show that $2.2(2)$ can not hold.

Assume, on the contrary, that there are $t \in T=\omega^{<\omega}$ and stationary subsets $D_{0}, D_{1}, \ldots, D_{|t|-1}$ of $\kappa$ such that for each $s \in\left(D_{0}, \ldots, D_{|t|-1}\right)$ we have

$$
A(s, t)=\emptyset \text {. }
$$

We can obviously assume that the sets $D_{i}$ are pairwise disjoint. Let $X_{i}=\bigcup\left\{A(\delta, t(i)): \delta \in D_{i}\right\}$ for every $i<|t|$. Then clearly $X_{i} \in \hat{\mathcal{A}}^{s}$ for every $i<|t|$, while $(+)$ implies $\bigcap_{i<k} X_{i}=\emptyset$, contradicting that $\hat{\mathcal{A}}^{s}$ is centered.

Definition 2.8. If $\kappa=\operatorname{cf}(\kappa)>\omega$, then principle $F^{s}(\kappa)(F(\kappa))$ is the following statement:

For every $T \subset \omega^{<\omega}$ and $\mathcal{A} \in \mathcal{M}(\kappa)$ (1) or (2) below holds:

(1) there is a stationary (cofinal) set $S \subset \kappa$ such that

$$
\mid\left\{A(s, t): t \in T \text { and } s \in(S)^{|t|}\right\} \mid \leq \omega .
$$

(2) there are $t \in T$ and stationary (cofinal) subsets $D_{0}, D_{1}, \ldots, D_{|t|-1}$ of $\kappa$ such that if $s_{0}, s_{1} \in\left(D_{0}, \ldots, D_{|t|-1}\right)$ with $s_{0}(i) \neq s_{1}(i)$ for each $i<|t|$ then we have

$$
A\left(s_{0}, t\right) \neq A\left(s_{1}, t\right) .
$$

Clearly, if $t, D_{0}, \ldots, D_{|t|-1}$ satisfy (2) then

$$
\left|\left\{A(s, t): s \in\left(D_{0}, \ldots, D_{|t|-1}\right)\right\}\right|=\kappa .
$$

There is a surprising connection between these principles and the dual versions $\hat{C}^{s}(\kappa)(\hat{C}(\kappa))$ of $C^{s}(\kappa)(C(\kappa))$, respectively. 
Theorem 2.9. $F^{s}(\kappa)(F(\kappa))$ implies $\hat{C}^{s}(\kappa)(\hat{C}(\kappa))$.

Proof. Let $\mathcal{A} \in \mathcal{M}(\kappa)$ and $T \subset \omega^{<\omega}$ and apply $F^{s}(\kappa)$ to $\mathcal{A}$ and $T$. Assume first that there is a stationary set $S \subset \kappa$ such that the family

$$
\mathcal{I}=\left\{A(s, t): t \in T \text { and } s \in(S)^{|t|}\right\}
$$

is countable.

Now for $t \in T, i<|t|$ and $I \in \mathcal{I} \cap[\omega]^{\omega}$ set

$$
D(I, t, i)=\{\alpha \in S: A(\alpha, t(i)) \supset I\} .
$$

If for some $t \in T$ and $I \in \mathcal{I} \cap[\omega]^{\omega}$ the set $D(I, t, i)$ is stationary for each $i<|t|$ then this $t$ and the sets $D(I, t, 0), \ldots, D(I, t,|t|-1)$ witness $2.3(2)$.

So we can assume that for all $t \in T$ and $I \in \mathcal{I} \cap[\omega]^{\omega}$ the set

$$
b(I, t)=\{i<|t|: D(I, t, i) \text { is non-stationary in } \kappa\}
$$

is not empty. Then the set

$$
D=\bigcup\left\{D(I, t, i): I \in \mathcal{I} \cap[\omega]^{\omega}, t \in T, i \in b(I, t)\right\}
$$

is not stationary and so $S^{\prime}=S \backslash D$ is stationary. We claim that $S^{\prime}$ witnesses 2.3(1). Assume on the contrary that $t \in T, s \in\left(S^{\prime}\right)^{|t|}$ and $I=A(s, t)$ is infinite. Then $I \in \mathcal{I} \cap[\omega]^{\omega}$ and $s(i) \in D(I, t, i)$ for each $i<|t|$. Since $s(i) \notin D$ it follows that $D(I, t, i)$ is stationary for each $i<|t|$, that is, $b(I, t)=\emptyset$, which is a contradiction.

Assume now that there are $t \in T$ and stationary subsets $D_{0}, D_{1}$, $\ldots, D_{|t|-1}$ of $\kappa$ such that if $s_{0}, s_{1} \in\left(D_{0}, \ldots, D_{|t|-1}\right)$ with $s_{0}(i) \neq s_{1}(i)$ for each $i<|t|$ then

$$
A\left(s_{0}, t\right) \neq A\left(s_{1}, t\right) .
$$

We show that in this case again 2.3(2) holds. Indeed, for each $I \in[\omega]^{<\omega}$ pick $s_{I} \in\left(D_{0}, \ldots, D_{|t|-1}\right)$ such that $A\left(s_{I}, t\right)=I$ provided that there is such an $s$. Let $R=\bigcup\left\{s_{I}(i): I \in[\omega]^{<\omega}, i<|t|\right\}$ and $D_{i}^{\prime}=D_{i} \backslash R$ for $i<|t|$. Now if $s \in\left(D_{0}^{\prime}, \ldots, D_{|t|-1}^{\prime}\right)$ then for any $I \in[\omega]^{<\omega}$ we have $s_{I}(i) \neq s(i)$ for each $i<|t|$, hence $I=A\left(s_{I}, t\right) \neq A(s, t)$. As $I$ was an arbitrary element of $[\omega]^{<\omega}$ we conclude that $|A(s, t)|=\omega$.

If $\kappa=\operatorname{cf}(\kappa)>c$ then $C^{s}(\kappa)$ and $F^{s}(\kappa)$ are trivially valid. Indeed given $\mathcal{A} \in \mathcal{M}(\kappa)$ and $T \subset \omega^{<\omega}$ there is a stationary set $S \subset \kappa$ such that for any $\alpha, \beta \in S$ and $n \in \omega$ we have $A(\alpha, n)=A(\beta, n)$. Then $S$ witnesses 2.8(1) and so principle $F^{s}(\kappa)$ holds. If $S$ does not witness 
2.2 (1) then for some $t=\left\langle n_{0}, \ldots, n_{k-1}\right\rangle \in T$ we have $\bigcap_{i<k} A_{n_{i}}=\emptyset$. Thus $t$ and $D_{0}=D_{1}=\cdots=D_{k-1}=S$ witness 2.2. $(2)$.

As was mentioned in section 1, our principles are of interest only for $\kappa>\omega_{1}$. In fact, for $\kappa=\omega_{1}$, they are all false!

To see that $D\left(\omega_{1}\right)$ (so also $C\left(\omega_{1}\right)$ ) is false we may recall that in [7] we have constructed, in ZFC, a separable, first countable $P_{<\omega}$ space $X$ of size $\omega_{1}$. (A Hausdorff space $X$ is called $P_{<\omega}$ if the intersection of finitely many uncountable open subsets of $X$ is always non-empty.) We can assume that the underlying set of $X$ is $\omega_{1}$ and $\omega$ is dense in $X$. For each $\alpha<\omega_{1}$ let $\{U(\alpha, n): n<\omega\}$ be a neighbourhood base of $\alpha$ in $X$. Now consider the $\omega_{1} \times \omega$-matrix

$$
\mathcal{A}=\left\langle U(\alpha, n) \cap \omega: \alpha<\omega_{1}, n<\omega\right\rangle .
$$

Then $B \in \hat{\mathcal{A}}$ if and only if there is an uncountable open set $U \subset X$ such that $U \cap \omega \subset B$. Since $X$ is a $P_{<\omega}$ space it follows that $\hat{\mathcal{A}}$ is centered. But the space $X$ is Hausdorff, so there is not even a two element subset $S$ of $X$ such that $\mathcal{A}\lceil S$ is $\omega$-adic.

To show that $F\left(\omega_{1}\right)$ (and so also $\hat{C}\left(\omega_{1}\right)$ ) is false we need the following observation.

Theorem 2.10. There is a subfamily $\mathcal{A}=\left\{A_{\alpha}: \alpha<\omega_{1}\right\}$ of $[\omega]^{\omega}$ such that for any $n \in \omega$ and $I_{0}, \ldots, I_{n-1} \in\left[\omega_{1}\right]^{\omega_{1}}$ there are $\gamma_{i}, \delta_{i} \in I_{i}$ for $i<n$ with $\cap\left\{A_{\gamma_{i}}: i<n\right\}$ is infinite but $A_{\delta_{i}} \cap A_{\delta_{j}}$ is finite for any $i<j<n$.

Proof. The proof is based on two lemmas which are probably wellknown.

Lemma 2.11. There is a function $f:\left[\omega_{1}\right]^{2} \longrightarrow 2$ such that for any $n \in \omega$ and $I_{0}, \ldots, I_{n-1} \in\left[\omega_{1}\right]^{\omega_{1}}$ there are $\gamma_{i}, \delta_{i} \in I_{i}$ for $i<n$ such that $f\left(\gamma_{i}, \gamma_{j}\right)=0$ and $f\left(\delta_{i}, \delta_{j}\right)=1$ for each $i \neq j<n$.

Proof. We show that the Sierpienski coloring has this property. So let $\left\{r_{\alpha}: \alpha<\omega_{1}\right\}$ be pairwise different real numbers and for $\alpha<\beta<\omega_{1}$ put $f(\alpha, \beta)=0$ iff $r_{\alpha}<r_{\beta}$. Given $n \in \omega$ and $I_{0}, \ldots, I_{n-1} \in\left[\omega_{1}\right]^{\omega_{1}}$ let $x_{i}$ be a complete accumulation point of $A_{i}=\left\{r_{\alpha}: \alpha \in I_{i}\right\}$ with $x_{i} \neq x_{j}$ for $i<j<n$. We may assume that $x_{0}<x_{1}<\cdots<x_{n-1}$. So there are $I_{i}^{\prime} \in\left[I_{i}\right]^{\omega_{1}}$ for $i<n$ such that if $A_{i}^{\prime}=\left\{r_{\alpha}: \alpha \in I_{i}^{\prime}\right\}$ then $A_{i}^{\prime}<_{R} A_{j}^{\prime}$ whenever $i<j<n$. Now pick first $\gamma_{0} \in I_{0}^{\prime}$, then $\gamma_{1} \in I_{1}^{\prime} \backslash\left(\gamma_{0}+1\right)$, then $\gamma_{2} \in I_{2}^{\prime} \backslash\left(\gamma_{1}+1\right)$ and so on. Then we have $f\left(\gamma_{i}, \gamma_{j}\right)=0$ for each $i<j<n$. Next we pick $\delta_{n-1} \in I_{n-1}^{\prime}$, then $\delta_{n-2} \in I_{n-2}^{\prime} \backslash \delta_{n-1}$, 
then $\delta_{n-3} \in I_{n-3}^{\prime} \backslash \delta_{n-2}$ and so on. Then we have $f\left(\delta_{i}, \delta_{j}\right)=1$ for $i<j<n$.

A family $\mathcal{I} \subset \mathcal{P}\left(\omega_{1}\right)$ is called downwards closed if $\mathcal{P}(I) \subset \mathcal{I}$ for each $I \in \mathcal{I}$. Given a family $\mathcal{A}=\left\{A_{\alpha}: \alpha<\omega_{1}\right\} \subset \mathcal{P}(\omega)$ and $I, J \in\left[\omega_{1}\right]^{<\omega}$, let

$$
A[I, J]=\bigcap\left\{A_{\alpha}: \alpha \in I\right\} \backslash \bigcup\left\{A_{\beta}: \beta \in J\right\} .
$$

Put $A[I]=A[I, \emptyset]$.

Clearly, if $\mathcal{A} \subset \mathcal{P}(\omega)$ then $\left\{I \in[\omega]^{<\omega}:|A[I]|=\omega\right\}$ is downward closed. Our next result is a converse of this statement.

Lemma 2.12. If $\mathcal{I} \subset\left[\omega_{1}\right]^{<\omega}$ is downwards closed then there is a family $\mathcal{A}=\left\{A_{\alpha}: \alpha<\omega_{1}\right\}$ of subsets of $\omega$ such that

$$
\mathcal{I}=\left\{I \in\left[\omega_{1}\right]^{<\omega}: A[I] \text { is infinite }\right\}
$$

Proof. For $\alpha<\omega_{1}$ write $\mathcal{I}_{\alpha}=\mathcal{I} \cap[\alpha+1]^{<\omega}$.

We will define $A_{\alpha} \subset \omega$ by induction on $\alpha<\omega_{1}$ so as to satisfy the following inductive hypotheses $(\ddagger)_{\alpha}$ which is stronger than $(\dagger)$ restricted to $\alpha$ :

$$
\mathcal{I}_{\alpha}=\left\{I \in[\alpha+1]^{<\omega}: \forall J \in[\alpha \backslash I]^{<\omega} A[I, J] \text { is infinite. }\right\}
$$

The induction uses the following elementary fact.

Fact 2.13. If $\mathcal{B}$ and $\mathcal{D}$ are countable subfamilies of $[\omega]^{\omega}$ such that no element of $\mathcal{B}$ is covered by the union of finitely many elements of $\mathcal{D}$ then there is a set $X \subset \omega$ such that

(i) $B \cap X$ is infinite for each $B \in \mathcal{B}$,

(ii) $B \backslash X$ is infinite for each $B \in \mathcal{B}$,

(iii) $D \cap X$ is finite for each $D \in \mathcal{D}$.

Now, if $A_{\beta}$ has been defined and $\left(\ddagger_{\beta}\right)$ holds for all $\beta<\alpha$ then let $\mathcal{B}=\left\{A[I, J]: I \in \mathcal{I}_{\alpha}, J \in[\alpha]^{<\omega} \wedge I \cap J=\emptyset\right\}, \mathcal{D}=\{A[I, J]: I \in$ $\left.[\alpha]^{<\omega} \backslash \mathcal{I}_{\alpha}, J \in[\alpha]^{<\omega} \wedge I \cap J=\emptyset\right\}$ and apply fact 2.13 to get $A_{\alpha}$. It is easy to check that $\left(\ddagger_{\alpha}\right)$ will be satisfied.

To get a family $\mathcal{A}$ satisfying the requirements of theorem 2.10 take the function $f$ given by lemma 2.11 and apply lemma 2.12 to $\mathcal{I}=\{I \in$ $\left.\left[\omega_{1}\right]^{<\omega}: f^{\prime \prime} I \subset\{0\}\right\}$.

Theorem 2.10 yields immediately the following corollary: 
Corollary 2.14. There is a family $\mathcal{A}=\left\{A_{\alpha}: \alpha<\omega_{1}\right\}$ such that

$$
\hat{\mathcal{A}}=\left\{Y \subset \omega:\left|\left\{\alpha<\omega_{1}: A_{\alpha} \subset Y\right\}\right|=\omega_{1}\right\}
$$

is centered but for no $S \in\left[\omega_{1}\right]^{\omega_{1}}$ is $\left\{A_{\alpha}: \alpha \in S\right\}$ linked.

Let us remark that if $\mathcal{A}$ is almost disjoint then $\hat{\mathcal{A}}$ is centered if and only if $\mathcal{A}$ is a strong Luzin gap (i.e. there is no partition of $\omega$ into finitely many pieces such that each piece is almost disjoint to uncountably many elements of $\mathcal{A}$ ). If $\mathrm{MA}_{\omega_{1}}$ holds, then there is no strong Luzin gap (see [7, Theorem 3.2]), so in ZFC one can not construct an almost disjoint family $\mathcal{A}$ satisfying the requirements of corollary 2.14.

The family $\mathcal{A}$ of 2.14 can be used to give counterexamples to both $D\left(\omega_{1}\right)$ and $C\left(\omega_{1}\right)$, in fact via th same matrix in $\mathcal{M}\left(\omega_{1}\right)$.

Corollary 2.15. $\hat{C}\left(\omega_{1}\right)$ (and so $F\left(\omega_{1}\right)$ too) and $D\left(\omega_{1}\right)$ are both false.

Proof. Consider the family $\mathcal{A}=\left\{A_{\alpha}: \alpha<\omega_{1}\right\}$ given by 2.14. Put $T=\omega^{<\omega}$ and $A(\alpha, i)=A_{\alpha}$ for each $\alpha<\omega_{1}$ and $i<\omega$. Then neither 2.3[1] nor 2.3[2] can hold for $\mathcal{A}=\left\langle A(\alpha, i): \alpha<\omega_{1}, i<\omega\right\rangle$ and $T$. Moreover, the matrix $\mathcal{A}$ clearly contradicts $D\left(\omega_{1}\right)$.

\section{Consistency of the PRinciples in the Cohen model.}

A cardinal $\kappa$ is $\omega$-inaccessible if $\lambda^{\omega}<\kappa$ holds for each $\lambda<\kappa$. Given any set $I$ we denote by $\mathcal{C}_{I}$ the poset $\operatorname{Fn}(I, 2, \omega)$, i.e. the standard one adding $\kappa$ Cohen reals.

In this section we prove that if $\kappa$ is a regular $\omega$-inaccessible cardinal in some ground model $V$ and we add any number of Cohen reals to $V$ then in the extension the principles $C^{s}(\kappa), \hat{C}^{s}(\kappa)$ and $F^{s}(\kappa)$ are all satisfied. As we remarked in section 2 above the case $\kappa>\lambda$ is trivial, while the case $\kappa<\lambda$ can be reduced to the case $\kappa=\lambda$.

Since the proof of the latter is long and technical, we first sketch the main idea. So let us be given a matrix $\mathcal{A} \in \mathcal{M}(\kappa)$ and a set $T \subset \omega^{<\omega}$ in $V[G]$, where $G$ is $\mathcal{C}_{\kappa}$-generic over $V$. In the first part of the proof we find a set $I \in[\kappa]^{\omega}$ and a stationary set $S \subset \kappa$ such that in $V[G\lceil I]$ the sequences $\langle A(\alpha, i): i<\omega\rangle$ for $\alpha \in S$ have also pairwise isomorphic names with disjoint supports (contained in $\kappa \backslash I$ ). This reduction, carried out in lemma 3.6, will be the place where we use that $\kappa$ is regular and $\omega$-inaccessible in $\mathrm{V}$. In the second part of the proof, using slightly different arguments for $C^{s}(\kappa)$ and for $F^{s}(\kappa)$, we show that if some $\mathcal{A} \in \mathcal{M}(S)$ has names with these properties then either $S$ witnesses 2.2(1) (or 2.8(1), respectively) or some stationary sets $D_{i} \subset S$ witness 2.2(2) (or 2.8(2), respectively). In this second step we don't use that $\kappa$ is $\omega$-inaccessible or regular. 
In our forcing arguments we follow the notation of Kunen [11. Let us first recall definition [11, 5.11].

Definition 3.1. A $\mathcal{C}_{I}$-name $\dot{B}$ of a subset of some ordinal $\mu$ is called nice if for each $\nu<\mu$ there is an antichain $B_{\nu} \subset \mathcal{C}_{I}$ such that

$$
\dot{B}=\left\{\langle p, \hat{\nu}\rangle: \nu \in \mu \wedge p \in B_{\nu}\right\}=\bigcup\left\{B_{\nu} \times\{\hat{\nu}\}: \nu \in \mu\right\} .
$$

We let $\operatorname{supp}(\dot{B})=\bigcup\left\{\operatorname{dom}(p): p \in \bigcup_{\nu<\mu} B_{\nu}\right\}$.

It is well-known (see e.g. lemma [11, 5.12]) that every set of ordinals in $V[G]$ has a nice name in $V$.

If $\varphi$ is a bijection between two sets $I$ and $J$ then $\varphi$ lifts to a natural isomorphism between $\mathcal{C}_{I}$ and $\mathcal{C}_{J}$, which will be also denoted by $\varphi$, as follows: for $p \in \mathcal{C}_{I}$ let $\operatorname{dom}(\varphi(p))=\varphi^{\prime \prime} \operatorname{dom}(p)$ and $\varphi(p)(\varphi(\xi))=p(\xi)$. Moreover $\varphi$ also generates a bijection between the nice $\mathcal{C}_{I}$-names and the nice $\mathcal{C}_{J}$-names $\left(\right.$ see $[11,7.12]$ ): if $\dot{B}$ is a nice $\mathcal{C}_{I}$-name then let $\varphi(\dot{B})=\{\langle\varphi(p), \hat{\xi}\rangle:\langle p, \hat{\xi}\rangle \in \dot{B}\}$. If $I$ and $J$ are sets of ordinals with the same order type then $\varphi_{I, J}$ is the natural order-preserving bijection from $I$ onto $J$.

Definition 3.2. Assume $I, J \subset \kappa$, moreover $\dot{A}_{i}$ and $\dot{B}_{i}$ are nice $\mathcal{C}_{\kappa^{-}}$ names of subsets of $\omega$ for $i<\omega$, such that $\operatorname{supp}\left(\dot{A}_{i}\right) \subset I$ and $\operatorname{supp}\left(\dot{B}_{i}\right) \subset$ $J$. We say that the structures of names $\left\langle I, \dot{A}_{i}: i<\omega\right\rangle$ and $\left\langle J, \dot{B}_{i}: i<\omega\right\rangle$ are twins if $I$ and $J$ have the same order type and

(1) $\varphi_{I, J}$ is the identity on $I \cap J$,

(2) $\varphi_{I, J}\left(\dot{A}_{i}\right)=\dot{B}_{i}$ for each $i<\omega$.

Definition 3.3. Assume that $I \subset \kappa, G$ is a $\mathcal{C}_{\kappa}$-generic filter over $V$ and $H=G\left\lceil I\right.$. If $\dot{B}$ is a nice $\mathcal{C}_{\kappa}$-name of a subset of some ordinal $\mu$ we define in $V[H]$ the $\mathcal{C}_{\kappa \backslash I}$ name $\pi^{H}(\dot{B})$ as follows:

$$
\pi^{H}(\dot{B})=\{\langle p\lceil\kappa \backslash I, \hat{\nu}\rangle:\langle p, \hat{\nu}\rangle \in \dot{B} \wedge p\lceil I \in H\} .
$$

Lemma 3.4. $\pi^{H}(\dot{B})$ is a nice $\mathcal{C}_{\kappa \backslash I}$-name in $V[H]$ and $\operatorname{supp}\left(\pi^{H}(\dot{B})\right) \subset$ $\operatorname{supp}(\dot{B}) \backslash I$, moreover

$$
\operatorname{val}\left(\pi^{H}(\dot{B}), G\lceil(\kappa \backslash I))=\operatorname{val}(\dot{B}, G) .\right.
$$

Proof. Straightforward from the construction.

Definition 3.5. Assume that $S \subset \kappa$. A matrix $\dot{\mathcal{B}}=\langle\dot{B}(\alpha, i): \alpha \in S, i<\omega\rangle$ of nice $\mathcal{C}_{\kappa}$-names of subsets of $\omega$ is called a nice $S$-matrix if conditions (i) and (ii) below hold: 
(i) putting $J_{\alpha}=\bigcup_{i<\omega} \operatorname{supp}(\dot{B}(\alpha, i))$ the sets $\left\{J_{\alpha}: \alpha \in S\right\}$ are pairwise disjoint,

(ii) the structures of names $\left\{\left\langle J_{\alpha}, \dot{B}(\alpha, i): i<\omega\right\rangle: \alpha \in S\right\}$ are pairwise twins.

We denote by $\mathcal{N}(S)$ the family of nice $S$-matrices.

Lemma 3.6. (Reduction lemma) Assume that $\kappa$ is a regular, $\omega$ inaccessible cardinal, $G$ is $\mathcal{C}_{\kappa}$-generic over $V$ and $\mathcal{A} \in \mathcal{M}(\kappa)$ in $V[G]$. Then there are a countable set $I \subset \kappa$ and a stationary set $S \subset \kappa$ in $V$ such that, in $V[G\lceil I]$, there is $\mathcal{B} \in \mathcal{N}(S)$ satisfying $V[G] \models " A(\alpha, i)=$ $\operatorname{val}(\dot{B}(\alpha, i), G\lceil(\kappa \backslash I))$ " for each $\alpha \in S$ and $i \in \omega$.

Proof. Assume that

$$
1_{\mathcal{C}_{\kappa}} \Vdash " \dot{\mathcal{A}}=\langle\dot{A}(\alpha, i): \alpha<\kappa, i<\omega\rangle \in \mathcal{M}(\kappa) . "
$$

We can assume that all the names $\dot{A}(i, \alpha)$ are nice. Let $I_{\alpha}=\bigcup_{i<\omega} \operatorname{supp}(\dot{A}(\alpha, i))$.

We need a strong version of Erdős-Rado $\Delta$-system theorem saying that there is a stationary set $T \subset \kappa$ such that $\left\{I_{\alpha}: \alpha \in T\right\}$ forms a $\Delta$ system with some kernel $I$, moreover sup $I<\min I_{\alpha} \backslash I$ for each $\alpha \in T$. Although this statement is well-known we present a proof because we could not find any reference to it.

Erdös-Rado Theorem . If $\kappa$ is an $\omega$-inaccessible regular cardinal and $\mathcal{X}=\left\{X_{\alpha}: \alpha<\kappa\right\}$ is a family of countable sets then there is a stationary set $I \subset \kappa$ such that $\left\{X_{\alpha}: \alpha \in I\right\}$ forms a $\Delta$-system.

Proof. Since $|\cup \mathcal{A}| \leq \kappa$ we can assume that $X_{\alpha} \subset \kappa$. Let $J=\{\alpha<$ $\left.\kappa: \operatorname{cf}(\alpha)=\omega_{1}\right\}$. Define the function $f: J \longrightarrow \kappa$ by the stipulation $f(\alpha)=\sup \left(X_{\alpha} \cap A\right)$. Since $X_{\alpha}$ is countable and $\operatorname{cf}(\alpha)=\omega_{1}$ we have $f(\alpha)<\alpha$, i.e. the function $f$ is regressive on the stationary set $J$. So by the Fodor lemma, $f$ is constant on a stationary set $K \subset J$. Say $f^{\prime \prime} K=\{\nu\}$. For $\alpha \in K$ let $h(\alpha)=X_{\alpha} \cap \nu$. Since $\nu<\kappa$ it follows that the range of $h$ is of size $|\nu|^{\omega}<\kappa$. But $K$ is stationary, so there is a stationary $M \subset K$ such that $h$ is constant on $M$, say $h^{\prime \prime} M=\{A\}$.

For $\alpha \in \kappa$ let $g(\alpha)=\sup X_{\alpha}$. Then the set

$$
C=\{\beta<\kappa: g(\gamma)<\beta \text { for each } \gamma<\beta\}
$$

is club in $\kappa$. Let $I=M \cap C$. We show that $\left\{X_{\alpha}: \alpha \in I\right\}$ forms a $\Delta$-system with kernel $A$. Let $\alpha, \beta \in I, \alpha<\beta$. Then $X_{\alpha} \cap X_{\beta}=$ $\left(X_{\alpha} \cap\left(X_{\beta} \cap \beta\right)\right) \cup\left(X_{\alpha} \cap\left(X_{\beta} \backslash \beta\right)\right.$.

But $\left(X_{\beta} \cap \beta\right)=A$ and so $\left(X_{\alpha} \cap\left(X_{\beta} \cap \beta\right)\right)=A$. Since $\beta \in C$ it follows that $g(\alpha)<\beta$, i.e. $X_{\alpha} \subset \beta$ and so $\left(X_{\alpha} \cap\left(X_{\beta} \backslash \beta\right)=\emptyset\right.$. Putting together these two equations we obtain $X_{\alpha} \cap X_{\beta}=A$ which was to be proved. 
Since $2^{\omega}<\kappa=\operatorname{cf}(\kappa)$ and there are only $2^{\omega}$ different isomorphism types of structures of names there is a stationary set $S \subset T$ such that the structures of names $\left\{\left\langle I_{\alpha}, \dot{A}(\alpha, i): i<\omega\right\rangle: \alpha \in S\right\}$ are pairwise twins.

From now on we work in $V\left[G\lceil I]\right.$. Let $\dot{B}(\alpha, i)=\pi^{G\lceil I}(\dot{A}(\alpha, i))$ for $\alpha \in S$ and $i \in \omega$. Then $\operatorname{supp}(\dot{B}(\alpha, i)) \subset J_{\alpha}=I_{\alpha} \backslash I$ and the structures of names $\left\langle J_{\alpha}, \dot{B}(\alpha, i): i<\omega\right\rangle$ are pairwise twins by lemma 3.4 above. Thus $\mathcal{B}=\langle\dot{B}(\alpha, i): \alpha \in S, i<\omega\rangle \in \mathcal{N}(S)$.

Definition 3.7. Assume that $S \subset \kappa$. A sequence $\dot{\mathcal{B}}=\left\langle\left\langle J_{\alpha}, \dot{B}_{\alpha}\right\rangle: \alpha \in S\right\rangle$ is called a nice $S$-sequence if conditions (i) and (ii) below hold:

(i) $J_{\alpha} \in[\kappa]^{\omega}, \dot{B}_{\alpha}$ is a nice $\mathcal{C}_{J_{\alpha}}$-name, and $J_{\alpha}$ for $\alpha \in S$ are pairwise disjoint,

(ii) the structures of names $\left\langle J_{\alpha}, \dot{B}_{\alpha}\right\rangle$ for $\alpha \in S$ are pairwise twins.

We denote by $\mathcal{S}(S)$ the family of nice $S$-sequences.

Lemma 3.8. (Homogeneity lemma) Assume that $S \subset \kappa$ and $\dot{\mathcal{B}}=$ $\left\langle\left\langle J_{\alpha}, \dot{B}_{\alpha}\right\rangle: \alpha \in S\right\rangle$ is a nice $S$-sequence. If $\varphi\left(x_{0}, x_{1}, \ldots, x_{n-1}, z\right)$ is a formula with free variables $x_{0}, x_{1}, \ldots, x_{n-1}, z$ and $Z$ is an element of the ground model, then (1) or (2) below holds:

(1) $1_{\mathcal{C}_{\kappa}} \|-“ \varphi\left(\dot{B}_{s(0)} \dot{B}_{s(1)}, \ldots, \dot{B}_{s(n-1)}, \hat{Z}\right)$ for all $s \in(S)^{n}$ ",

(2) for some $r \in \mathcal{C}_{\kappa}$ we have $r \|$ " there are subsets $\dot{D}_{0}, \dot{D}_{1}, \ldots, \dot{D}_{n-1}$ of $S$ such that

(a) for each $i<n$ and $A \in[S]^{\omega} \cap V$ we have $\dot{D}_{i} \cap A \neq \emptyset$,

(b) $\neg \varphi\left(\dot{B}_{s(0)} \dot{B}_{s(1)}, \ldots, \dot{B}_{s(n-1)}, \hat{Z}\right)$ for all $s \in\left(\dot{D}_{0}, \dot{D}_{1}, \ldots, \dot{D}_{k-1}\right)$ ".

Proof. Assume that (1) fails, that is, there are $p \in \mathcal{C}_{\kappa}$ and $s \in(S)^{k}$ such that

$$
p \Vdash " \neg \varphi\left(\dot{B}_{s(0)}, \ldots, \dot{B}_{s(n-1)}, \hat{Z}\right) " .
$$

Let $J=\bigcup_{i<k} J_{s(i)}$ and $p^{\prime}=p\left\lceil J\right.$ and $r=p \backslash p^{\prime}$. Since the sets $J_{\alpha}$ are pairwise disjoint we can assume that $\operatorname{dom}(r) \cap J_{\alpha}=\emptyset$ for each $\alpha \in S$.

For $\langle\alpha, \beta\rangle \in S^{2}$ we denote by $\varphi_{\alpha, \beta}$ the natural order preserving bijection between $J_{\alpha}$ and $J_{\beta}$. For $\beta \in S$ and $i<k$ let $p(\beta, i)=$ $\varphi_{s(i), \beta}\left(p\left\lceil J_{s(i)}\right)\right.$. For $i<k$ define the $\mathcal{C}_{\kappa}$-name $\dot{D}_{i}$ of a subset of $S$ as follows: $\dot{D}_{i}=\{\langle p(\beta, i), \hat{\beta}\rangle: \beta \in S\}$. Then

$$
V[G] \models " D_{i}=\operatorname{val}\left(\dot{D}_{i}, G\right)=\{\beta \in S: p(\beta, i) \in G\} ",
$$


where $G$ is $\mathcal{C}_{\kappa}$-generic over $V$. Since the supports of $p(\beta, i)$ for $\beta \in S$ are pairwise disjoint a standard density argument gives that $D_{i} \cap A \neq \emptyset$ whenever $A \in[S]^{\omega} \cap V$, hence (a) holds.

To show (b) assume that $r \in G$ and

$$
V[G] \models u \in\left(D_{0}, \ldots, D_{k-1}\right) .
$$

Since $u$ is finite we have $u \in V$. Let $J^{*}=\bigcup_{i<k} J_{u(i)}$ and $\psi=\bigcup_{i<k} \varphi_{u(i), s(i)}$. Then $\psi$ is a bijection between $J^{*}$ and $J$ and so it extends to isomorphisms between $\mathcal{C}_{J^{*}}$ and $\mathcal{C}_{J}$, and between the families of nice $\mathcal{C}_{J^{*}}$-names and of nice $\mathcal{C}_{J}$-names. Let $\Psi$ be the natural extension of $\psi$ to a permutation of $\kappa$ :

$$
\Psi(\nu)= \begin{cases}\psi(\nu) & \text { if } \nu \in J^{*}, \\ \psi^{-1}(\nu) & \text { if } \nu \in J, \\ \nu & \text { if } \nu \in \kappa \backslash\left(J \cup J^{*}\right) .\end{cases}
$$

Then $\Psi$ extends to an automorphism of $\mathcal{C}_{\kappa}$, and also to an automorphism of nice $\mathcal{C}_{\kappa}$-names. Clearly if $q \in \mathcal{C}_{J^{*}}$ and $\dot{B}$ is a nice $\mathcal{C}_{J^{*}}$-name then $\psi(q)=\Psi(q)$ and $\psi(\dot{B})=\Psi(\dot{B})$. Observe that $\Psi(r)=r$ and $\Psi(\hat{Z})=\hat{Z}$.

Let $G^{*}=\Psi^{\prime \prime} G$. Then $G^{*}$ is also a $\mathcal{C}_{\kappa}$-generic filter over $V$ and since $\Psi\left(\dot{B}_{u(i)}\right)=\dot{B}_{s(i)}$ it follows that

$$
\operatorname{val}\left(\dot{B}_{u(i)}, G\right)=\operatorname{val}\left(\dot{B}_{s(i)}, G^{*}\right) .
$$

But $p(u(i), i) \in G$, so $p\left\lceil J_{s(i)}=\psi(p(u(i), i)) \in G^{*}\right.$. Thus $p=r \cup$ $\bigcup_{i<k} p\left\lceil J_{s(i)} \in G^{*}\right.$ as well. Since $p \Vdash \neg \varphi\left(\dot{B}_{s(0)}, \ldots, \dot{B}_{s(n-1)}, \hat{Z}\right)$ and so $V\left[G^{*}\right] \models " \neg \varphi\left(B_{s(0)}, \ldots, B_{s(n-1)}, Z\right)$ ", by $(\bullet)$ this implies

$$
V[G] \models " \neg \varphi\left(B_{u(0)}, \ldots, B_{u(n-1)}, Z\right) "
$$

which was to be proved.

Theorem 3.9. If $\kappa$ is a regular, $\omega$-inaccessible cardinal then for each cardinal $\lambda$ we have

$$
V^{\mathcal{C}_{\lambda}} \models C^{s}(\kappa) \text { and } \hat{C}^{s}(\kappa) \text { hold } .
$$

Proof. We deal only with $C^{s}(\kappa)$ because the same argument works for $\hat{C}^{s}(\kappa)$. As we observed in section $⿴$ we can assume that $\kappa \leq \lambda$. First we investigate the case $\lambda=\kappa$.

Assume that

$$
1_{\mathcal{C}_{\kappa} \Vdash} \Vdash \dot{\mathcal{A}}=\langle\dot{A}(\alpha, i): \alpha<\kappa ; i<\omega\rangle \in \mathcal{M}(\kappa) \text { and } T \subset \omega^{<\omega} . "
$$


Applying the reduction lemma 3.6 and that $T$ is countable we can find a countable set $I \subset \kappa$ and a stationary set $S \subset \kappa$ in $V$ and a nice $S$-matrix $\mathcal{B}$ in $V[G\lceil I]$ such that

$$
V[G] \models " \operatorname{val}(\dot{A}(\alpha, i), G)=\operatorname{val}(\dot{B}(\alpha, i), G\lceil(\kappa \backslash I)) "
$$

for $\alpha \in S$ and $i \in \omega$, moreover $T \in V[G\lceil I]$.

We show that for each $q \in \mathcal{C}_{\kappa}$ there is a condition $r \leq q$ in $\mathcal{C}_{\kappa}$ such that $r \Vdash$ "2.2(1) or 2.2(2) holds". Let $I^{\prime}=I \cup \operatorname{dom}(q)$.

For each $t \in T$ let $\varphi_{t}\left(x_{0}, \ldots, x_{|t|-1}\right)$ be the following formula:

$$
\varphi\left(\left\langle B_{0, k}: k<\omega\right\rangle, \ldots,\left\langle B_{|t|-1, k}: k<\omega\right\rangle\right) \Longleftrightarrow \bigcap_{i<|t|} B_{i, t(i)} \neq \emptyset .
$$

Applying the homogeneity lemma 3.8 to $V\left[G\left\lceil I^{\prime}\right]\right.$ as our ground model and to every $\varphi_{t}$ we get that either $q \Vdash$ '2.2(1) holds" or $q \cup p \Vdash$ "2.2(2) holds". Let us remark that $3.8(2)$ (a) implies that as $S$ is stationary, so is each $D_{i}$.

Thus we have proved the theorem in the case $\kappa=\lambda$. If $\lambda>\kappa$ and $\mathcal{A} \in(\mathcal{M}(\kappa))^{V[G]}$, where $G$ is $\mathcal{C}_{\lambda}$-generic over $V$, then there is $J \in[\lambda]^{\kappa}$ such that $\mathcal{A} \in V[G[J]$. The stationary sets that witness 2.2(1) or $2.2(2)$ in $V[G\lceil J]$ remain stationary in $V[G]$, and so we are done.

Theorem 3.10. If $\kappa$ is a regular, $\omega$-inaccessible cardinal then for each cardinal $\lambda$ we have

$$
V^{\mathcal{C}_{\lambda}} \models F^{s}(\kappa) \text { holds. }
$$

Proof. As in 3.9 the important case is when $\lambda=\kappa$, because the case $\lambda<\kappa$ is trivial and the case $\kappa<\lambda$ can be reduced to the case $\kappa=\lambda$.

So assume that

$$
1_{\mathcal{C}_{\kappa}} \Vdash " \dot{\mathcal{A}}=\langle\dot{A}(\alpha, i): \alpha<\kappa, i<\omega\rangle \in \mathcal{M}(\kappa) . "
$$

Applying lemma 3.6 we can find a countable set $I \subset \kappa$, a stationary set $S \subset \kappa$ in $V$ and in $V[G\lceil I]$ a nice $S$-matrix $\mathcal{B}$ such that $V[G] \models$ " $A(\alpha, i)=\operatorname{val}(\dot{B}(\alpha, i), G\lceil(\kappa \backslash I))$ " for each $\alpha \in S$ and $i \in \omega$, moreover $T \in V[G\lceil I]$.

We need the following lemma that is probably well-known.

Lemma 3.11. If $H$ is a $\mathcal{C}_{\kappa}$-generic filter over $V$ and $I$, $J$ are disjoint subsets of $\kappa$ then

$$
V[H] \models " \mathcal{P}(\omega) \cap V[H\lceil I] \cap V[H\lceil J]=\mathcal{P}(\omega) \cap V . "
$$

Proof of lemma. Assume that $\dot{A}$ is a nice $\mathcal{C}_{I}$-name, $\dot{B}$ is a nice $\mathcal{C}_{J}$-name, $p \in \mathcal{C}_{\kappa}$ and $p \Vdash " \dot{A}=\dot{B} "$. We can assume that $\operatorname{dom}(p) \subset I \cup J$. We show that for each $n \in \omega$ we have that $p\lceil I \Vdash " n \in \dot{A} "$ or $p\lceil I \Vdash$ 
" $\hat{n} \notin \dot{A}$ ". Indeed, if $p\lceil I \Vdash / n$ " $\hat{n} \in \dot{A}$ " then there is a condition $q \leq p\lceil I$ in $\mathcal{C}_{I}$ such that $q \Vdash$ " $\hat{n} \notin \dot{A}$ " and so $p \cup q \Vdash$ " $\hat{n} \notin \dot{B}$ ". But $\dot{B}$ is a $\mathcal{C}_{J}$ name so $(p \cup q)\lceil J=p\lceil J$ forces the same statement, $p\lceil J \Vdash " \hat{n} \notin \dot{B}$ ". But $\Vdash \dot{A}=\dot{B}$ and so $p \Vdash \hat{n} \notin \dot{A}$ as well. Thus $p$ decides the elements of $\dot{A}$, in other words, $p \Vdash " \dot{A} \in V$ ".

To conclude the proof we show that if $q \in \mathcal{C}_{\kappa}$ then there is a condition $r \leq q$ in $\mathcal{C}_{\kappa}$ such that $r \Vdash$ 2.8(1) or 2.8(2) holds". Let $I^{\prime}=I \cup \operatorname{dom}(q)$.

For each $t \in T$ let $\varphi_{t}\left(x_{0}, \ldots, x_{|t|-1}\right)$ be the following formula:

$$
\varphi\left(\left\langle B_{0, k}: k<\omega\right\rangle, \ldots,\left\langle B_{|t|-1, k}: k<\omega\right\rangle\right) \Longleftrightarrow \bigcap_{i<|t|} B_{i, t(i)} \in(\mathcal{P}(\omega))^{V} .
$$

Applying the homogeneity lemma 3.8 to $V\left[G\left\lceil I^{\prime}\right]\right.$ as our ground model we get that $(\mathrm{A})$ or $(\mathrm{B})$ below holds:

(A) $1_{\mathcal{C}_{\kappa}} \Vdash " B(s, t) \in(\mathcal{P}(\omega))^{V}$ for each $t \in T$ and $s \in(S)^{|t|}$,"

(B) for some $t \in T$ and $p \in \mathcal{C}_{\kappa}$ we have $p \Vdash$ " there are subsets $\dot{D}_{0}, \dot{D}_{1}, \ldots, \dot{D}_{|t|-1}$ of $S$ such that

(a) for each $A \in[S]^{\omega} \cap V$ we have $A \cap \dot{D}_{i} \neq \emptyset$ for each $i<|t|$,

(b) If $s \in\left(\dot{D}_{0}, \dot{D}_{1}, \ldots, \dot{D}_{|t|-1}\right)$ we have

$$
\dot{B}(s, t) \notin(\mathcal{P}(\omega))^{V} . "
$$

Let $J_{\alpha}=\bigcup_{i<\omega} \operatorname{supp}(\dot{B}(\alpha, i))$ for $\alpha \in S$ and denote by $\varphi_{\alpha, \beta}$ the natural order preserving bijection between $J_{\alpha}$ and $J_{\beta}$ for $\langle\alpha, \beta\rangle \in S^{2}$.

Assume first that (A) holds. Fix $t \in T$ and $s \in(S)^{|t|}$. Write $\alpha_{i}=s(i)$ for $i<|t|$. Since $\mathcal{C}_{\kappa}$ is c.c.c, there is in $V$ a countable set $\mathcal{I}_{t} \subset \mathcal{P}(\omega)$ such that $1_{\mathcal{C}_{\kappa}} \Vdash " ~ " \cap \dot{B}\left(\alpha_{i}, n_{i}\right) \in \mathcal{I}_{t}$ ".

Assume that $\left\langle\delta_{0}, \ldots, \delta_{|t|-1}\right\rangle \in(S)^{k}$.

Let $J^{*}=\bigcup_{i<k} J_{\delta_{i}}, J=\bigcup_{i<k} J_{\alpha_{i}}$, and $\psi=\bigcup_{i<k} \varphi_{\delta_{i}, \alpha_{i}}$. Then $\psi$ is a bijection between $J^{*}$ and $J$ and so it lifts up to an isomorphism between $\mathcal{C}_{J^{*}}$ and $\mathcal{C}_{J}$ and between the families of nice $\mathcal{C}_{J^{*}}$-names and nice $\mathcal{C}_{J}$-names.

Let $G$ be $\mathcal{C}_{\kappa}$-generic and put $G_{0}=G\left\lceil J^{*}\right.$. Since $\operatorname{supp}\left(\dot{B}\left(\delta_{i}, n_{i}\right)\right) \subset J^{*}$ it follows that $\operatorname{val}\left(\dot{B}\left(\delta_{i}, n_{i}\right), G\right)=\operatorname{val}\left(\dot{B}\left(\delta_{i}, n_{i}\right), G_{0}\right)$. Let $G_{1}=\psi^{\prime \prime} G_{0}$. Then $G_{1}$ is also a $\mathcal{C}_{J}$-generic filter and since $\psi\left(\dot{B}\left(\delta_{i}, n_{i}\right)\right)=\dot{B}\left(\alpha_{i}, n_{i}\right)$ it follows that

$$
\operatorname{val}\left(\dot{B}\left(\delta_{i}, n_{i}\right), G_{0}\right)=\operatorname{val}\left(\dot{B}\left(\alpha_{i}, n_{i}\right), G_{1}\right) .
$$

Since $1_{\mathcal{C}_{\kappa} \Vdash} \Vdash " \bigcap_{i<k} \dot{B}\left(\alpha_{i}, n_{i}\right) \in \mathcal{I}_{t}$ ", by $(\bullet)$ we have $1_{\mathcal{C}_{\kappa}} \Vdash " ~ " \bigcap \operatorname{val}\left(\dot{B}\left(\delta_{i}, n_{i}\right), G\right) \in$ $\mathcal{I}_{t}$ " as well. From this it is obvious that we have

$$
1_{\mathcal{C}_{\kappa}} \Vdash\left\{\dot{B}(t, s): t \in T \wedge s \in(S)^{|t|}\right\} \subset \mathcal{I}=\bigcup\left\{\mathcal{I}_{t}: t \in T\right\} .
$$


where $\mathcal{I}$ is countable as $T$ is.

Assume now that (A) fails and so (B) holds.

Let $G$ be $\mathcal{C}_{\kappa}$-generic with $p \in G$ and $\left\langle\gamma_{0}, \ldots, \gamma_{k-1}\right\rangle,\left\langle\delta_{0}, \ldots, \delta_{k-1}\right\rangle \in$ $\left(D_{0}, \ldots, D_{k-1}\right)$ such that

$V[G] \models$ " $\left\{\gamma_{i}, \delta_{i}\right\} \in\left[D_{i}\right]^{2}$ for $i<k$ are pairs of distict ordinals".

Let $J^{*}=\bigcup_{i<k} J_{\gamma_{i}}$ and $J^{\star}=\bigcup_{i<k} J_{\delta_{i}}$. Then $J^{*} \cap J^{\star}=\emptyset$, hence by lemma 3.11 we have $\mathcal{P}(\omega) \cap V\left[G\left\lceil J^{*}\right] \cap\left\lceil V\left[G\left\lceil J^{\star}\right]=\mathcal{P}(\omega) \cap V\right.\right.\right.$ and so $V[G] \models " \bigcap_{i<k} B\left(\delta_{i}, n_{i}\right) \notin V "$ implies that $V[G] \models " \bigcap_{i<k} B\left(\delta_{i}, n_{i}\right) \neq$ $\bigcap_{i<k} B\left(\gamma_{i}, n_{i}\right) "$

The theorem is proved.

\section{Applications}

We start with presenting some combinatorial applications because they are quite simple and so they nicely illustrate the use of our principles.

Kunen [12] proved that if one adds Cohen reals to a model of $C H$ then in the generic extension there is no strictly $C^{*}$-increasing chain of subsets of $\omega$ of length $\omega_{2}$. The first theorem we prove easily yields a corollary which is a generalization of Kunen's above result.

Theorem 4.1. If $C(\kappa)$ holds then for each $\mathcal{A} \subset[\omega]^{\omega}$ of size $\kappa$ either

(a) $\exists \mathcal{B} \in[\mathcal{A}]^{\kappa} \forall B \neq B^{\prime} \in \mathcal{B}\left|B \backslash B^{\prime}\right|=\omega$

or

(b) $\exists X \in[\omega]^{\omega}|\{A \in \mathcal{A}: A \subset X\}|=\left|\left\{A \in \mathcal{A}: X \subset^{*} A\right\}\right|=\kappa$.

Proof. Fix a $1-1$ enumeration $\left\{A_{\xi}: \xi<\kappa\right\}$ of $\mathcal{A}$. Let $A(\xi, 2 n)=A_{\xi} \backslash n$ and $A(\xi, 2 n+1)=\left(\omega \backslash A_{\xi}\right) \backslash n$. Put $T=\{\langle 2 i, 2 i+1\rangle: i \in \omega\}$. If $S \in[\kappa]^{\kappa}$ witnesses 2.2(1), then $\mathcal{B}=\left\{A_{\xi}: \xi \in S\right\}$ satisfies (a). If on the other hand $D, E \in[\kappa]^{\kappa}, D \cap E=\emptyset$, with $\langle 2 i, 2 i+1\rangle \in T$ show that 2.2[2] holds, then let $X=\cup\left\{A_{\xi}: \xi \in D\right\}$. Then $A_{\xi} \subset X$ for each $\xi \in D$ and $X \backslash i \subset A_{\zeta}$ for each $\zeta \in E$.

Corollary 4.2. If $C(\kappa)$ holds then there is no strictly $\subset^{*}$-increasing chain $\mathcal{T} \subset[\omega]^{\omega}$ of length $\kappa$.

The next theorem can be considered as a kind of dual to 4.1 .

Theorem 4.3. If $C(\kappa)$ holds then for each $\mathcal{A} \subset[\omega]^{\omega}$ of size $\kappa$ and for each natural number $k$ either 
(a) there is a family $\mathcal{B} \in[\mathcal{A}]^{\kappa}$ such that for each $\mathcal{B}^{\prime} \in[\mathcal{B}]^{k}$ we have $\left|\cap \mathcal{B}^{\prime}\right|=\omega$

or

(b) there are $k$ subfamilies $\mathcal{B}_{0}, \ldots, \mathcal{B}_{k-1}$ of $\mathcal{B}$ of size $\kappa$ such that

$$
\left|\bigcap_{i<k} \bigcup \mathcal{B}_{i}\right|<\omega
$$

Proof. Fix a $1-1$ enumeration $\left\{A_{\xi}: \xi<\kappa\right\}$ of $\mathcal{A}$. Let $A(\xi, n)=A_{\xi} \backslash n$ and put $T=\omega^{k}$. If $C \in[\kappa]^{\kappa}$ witnesses $2.2(1)$, then $\mathcal{B}=\left\{A_{\xi}: \xi \in C\right\}$ satisfies (a). If $D_{0}, \ldots, D_{k-1} \in[\kappa]^{\kappa}$ and $\left\langle n_{0}, \ldots, n_{k-1}\right\rangle \in T$ show that $2.2(2)$ holds, then we can assume that the $D_{i}$ are pairwise disjoint and if we set $\mathcal{B}_{i}=\left\{A_{\xi}: \xi \in D_{i}\right\}$ then we have

$$
\bigcap_{i<k} \bigcup \mathcal{B}_{i} \subset \max _{i<k} n_{i}
$$

Remark. In theorem 4.3 we can not replace (a) with the following (slightly stronger) condition (a'):

(a') there is a family $\mathcal{B} \in[\mathcal{A}]^{\kappa}$ such that for each $\mathcal{B}^{\prime} \in[\mathcal{B}]^{k+1}$ we have $\left|\cap \mathcal{B}^{\prime}\right|=\omega$

and if $k>2$ then (b) can not be replaced by

(b') there are pairwise disjoint subsets $X_{0}, \ldots, X_{k-1}$ of $\omega$ such that for each $i<k$ we have $\left\{A \in \mathcal{A}: A \subset^{*} X_{i}\right\} \neq \emptyset$,

because for each $k \in \omega$ one can construct in ZFC a family $\mathcal{A} \subset[\omega]^{\omega}$ of size $2^{\omega}$ such that $\cap \mathcal{A}^{\prime}$ is finite for every $\mathcal{A}^{\prime} \in[\mathcal{A}]^{k+1}$ but $\cap \mathcal{A}^{\prime}$ is infinite whenever $\mathcal{A}^{\prime} \in[\mathcal{A}]^{k}$. Indeed, let $T=2^{<\omega}$ be the Cantor tree, and for $n<\omega$ let $C_{n}=2^{n}$ be the $n^{\text {th }}$-level of $T$. For each $f \in 2^{\omega}$ let

$$
A(f)=\bigcup_{n<\omega}\left\{X \in\left[C_{n}\right]^{k}: f\lceil n \in X\}\right.
$$

and $\mathcal{A}=\left\{A(f): f \in 2^{\omega}\right\}$. If $\mathcal{B}^{\prime} \subset 2^{\omega}$ and $n<\omega$ then $\bigcap\{A(f)$ : $\left.f \in \mathcal{B}^{\prime}\right\} \cap\left[C_{n}\right]^{k} \neq \emptyset$ iff $\mid\left\{f\left\lceil n: f \in \mathcal{B}^{\prime}\right\} \mid \leq k\right.$. Thus $\mathcal{A}$ satisfies our requirements. This example is due to $\mathrm{A}$. Hajnal and included here with his kind permission.

Next we prove a consequence of theorem 4.3, but first we give a definition. 
Definition 4.4. Let $\kappa$ be a regular cardinal and $\mathcal{A} \subset[\omega]^{\omega}$ be an almost disjoint family. $\mathcal{A}$ is called a $\kappa$-Luzin gap if $|\mathcal{A}|=\kappa$ and there is no $X \in[\omega]^{\omega}$ such that both $\mid\{A \in \mathcal{A}:|A \backslash X|<\omega \mid\}=\kappa$ and $|\{A \in \mathcal{A}:|A \cap X|<\omega\}|=\kappa$. A Luzin-gap is an $\omega_{1}$-Luzin gap.

An $\omega_{1}$-Luzin gap can be constructed in ZFC and simple forcings give models in which there are $2^{\omega}$-Luzin gaps while $2^{\omega}$ is as large as you wish. The next corollary of theorem 4.3 implies that one can not construct $\omega_{2}$-Luzin gaps from the assumption $2^{\omega} \geq \omega_{2}$ alone .

Corollary 4.5. If $C(\kappa)$ holds then there is no $\kappa$-Luzin gap.

Proof. Assume that $\mathcal{A} \subset[\omega]^{\omega}$ is an almost disjoint family of size $\kappa$. Then we can not get a even a two element subfamily $\mathcal{B} \subset \mathcal{A}$ satisfying 4.3(a). So applying theorem 4.3 for this $\mathcal{A}$ and for $k=2$ there are subfamilies $\mathcal{B} \subset \mathcal{A}$ and $\mathcal{D} \subset \mathcal{A}$ of size $\kappa$ such that $(\cup \mathcal{B}) \cap(\cup \mathcal{D})$ is finite. Hence $X=\cup \mathcal{B}$ witnesses that $\mathcal{A}$ is not a $\kappa$-Luzin gap.

We have one more theorem of this type.

Theorem 4.6. If $C(\kappa)$ holds then for each $\mathcal{A} \subset[\omega]^{\omega}$ of size $\kappa$ either

(a) there is a centered subfamily $\mathcal{B} \subset \mathcal{A}$ of size $\kappa$,

or

(b) for some $k<\omega$ there are subfamilies $\mathcal{B}_{0}, \ldots, \mathcal{B}_{k-1}$ of $\mathcal{B}$ of size $\kappa$ such that

$$
\left|\bigcap_{i<k} \bigcup \mathcal{B}_{i}\right|<\omega
$$

Proof. We can argue as in the proof of theorem 4.3 using $T=\omega^{<\omega}$ instead of $T=\omega^{k}$.

For $\mathcal{A} \subset \mathcal{P}(\omega)$ and $\kappa<\omega$ let $\left.\mathcal{A}_{(k)}=\left\{\cap \mathcal{A}^{\prime}: \mathcal{A}^{\prime} \in[\mathcal{A}]^{n}\right\}\right\}$. Put $\mathcal{A}_{(<\omega)}=\bigcup_{k<\omega} \mathcal{A}_{(k)}$.

Theorem 4.7. If $F(\kappa)$ holds then for each family $\mathcal{A} \subset \mathcal{P}(\omega)$ of size $\kappa$ and for each natural number $k$ either

(a) $\left|\mathcal{A}_{(k)}\right|=\kappa$

or

(b) there is a subfamily $\mathcal{B} \subset \mathcal{A}$ of size $\kappa$ such that $\left|\mathcal{B}_{(k)}\right| \leq \omega$.

Proof. Fix a $1-1$ enumeration $\left\{A_{\alpha}: \alpha<\kappa\right\}$ of $\mathcal{A}$, let $T=\omega^{k}$ and consider the matrix $\mathcal{A}^{\prime}=\langle A(\alpha, n): \alpha<\kappa ; n<\omega\rangle \in \mathcal{M}(\kappa)$ defined by the stipulation $A(\alpha, n)=A_{\alpha}$. Apply $F(\kappa)$. If 2.8(2) holds, then $\left|\mathcal{A}_{(k)}\right|=\kappa$. If $S \in[\kappa]^{\kappa}$ witnesses $2.8(1)$ then subfamily $\mathcal{B}=\left\{A_{\alpha}: \alpha \in\right.$ $S\}$ satisfies $\left|\mathcal{B}_{(k)}\right|=\omega$. 
Theorem 4.8. If $F(\kappa)$ holds then for each family $\mathcal{A} \subset \mathcal{P}(\omega)$ of size $\kappa$ either

(a) there is a natural number $k$ such that $\left|\mathcal{A}_{(k)}\right|=\kappa$

or

(b) there is a subfamily $\mathcal{B} \subset \mathcal{A}$ of size $\kappa$ such that $\left|\mathcal{B}_{(<\omega)}\right| \leq \omega$.

Proof. We can argue as in the proof of theorem 4.7 using $T=\omega^{<\omega}$ instead of $T=\omega^{k}$.

Now we turn to applying our principles to topology. We start with an application of the relatively weak principle $D(\kappa)$.

A. Dow [2] proved that if we add $\omega_{2}$ Cohen reals to a model of $G C H$ then in the generic extension $\beta \omega$ can be embedded into every separable, compact $T_{2}$ space of size $>c=\omega_{2}$. Here we show that $c=\omega_{2}=2^{\omega_{1}}$ together with $D\left(\omega_{2}\right)$ suffice to imply this statement.

First we need a lemma based on the observation that large separable spaces contain many "similar" points.

Given a topological space $X$ and a point $x \in X$ we denote by $\mathcal{V}_{X}(x)$ the neighbourhood filter of $x$ in $X$, that is, $\mathcal{V}_{X}(x)=\{U \subset X: x \in$ $\left.\operatorname{int}_{X}(U)\right\}$. If $D$ is a dense subset of $X$ let $\mathcal{V}_{X}(x)\lceil D=\{U \cap D: D \in$ $\left.\mathcal{V}_{X}(x)\right\}$. We omit the subscript $X$ if it may not cause any confusion.

In section 2 we defined the operation $\hat{\mathcal{A}}$ for $\mathcal{A} \in \mathcal{M}(\kappa)$. By an abuse of notation we define $\hat{\mathcal{A}}$ for every family $\mathcal{A}$ of subsets of $\omega$ as follow:

$$
\hat{\mathcal{A}}=\{X \subset \omega:|\mathcal{A} \cap P(X)|=|\mathcal{A}|\} .
$$

Lemma 4.9. Assume that $X$ is a separable regular topological space of size $>c^{<c}$, where $c=2^{\omega}, D \in[X]^{\omega}, \bar{D}=X$. Then there are a point $x \in X$ and a family $\mathcal{A}=\left\{A_{\alpha}, B_{\alpha}: \alpha<c\right\} \subset \mathcal{P}(D)$ such that

(1) $\overline{A_{\alpha}} \cap \overline{B_{\alpha}}=\emptyset$ for each $\alpha<c$,

(2) $\hat{\mathcal{A}} \subset \mathcal{V}(x)\lceil D$.

Proof. Fix an enumeration $\left\{D_{\xi}: \xi<c\right\}$ of $\mathcal{P}(D)$ and let $\mathcal{D}_{\alpha}=\left\{D_{\xi}\right.$ : $\xi<\alpha\}$ for $\alpha<c$. For $x \in X$ and $\alpha<c$ let $\mathcal{V}(x, \alpha)=\left(\mathcal{V}(x)\lceil D) \cap \mathcal{D}_{\alpha}\right.$. A point $x \in X$ is called special if there is an $\alpha<c$ such that $\mathcal{V}(x, \alpha) \neq$ $\mathcal{V}(y, \alpha)$ for each $y \in X \backslash\{x\}$. Clearly there are at most $c^{<c}$ special points in $X$. Since $|X|>c^{<c}$ we can pick a point $x \in X$ which is not special. Then for each $\alpha<c$ we can find a point $x_{\alpha} \neq x$ in $X$ such that $\mathcal{V}\left(x_{\alpha}, \alpha\right)=\mathcal{V}(x, \alpha)$. Since $X$ is regular the points $x$ and $x_{\alpha}$ have neighbourhoods $U_{\alpha}$ and $W_{\alpha}$, respectively, with $\overline{U_{\alpha}} \cap \overline{W_{\alpha}}=\emptyset$. Let $A_{\alpha}=U_{\alpha} \cap D$ and $B_{\alpha}=W_{\alpha} \cap D$.

Now assume that $E \in \hat{\mathcal{A}}$ and pick $\xi<c$ with $E=D_{\xi}$. We can find $\alpha<c$ such that $\xi<\alpha$ and either $A_{\alpha} \subset E$ or $B_{\alpha} \subset E$. Hence 
$E \in \mathcal{V}(x, \alpha) \cup \mathcal{V}\left(x_{\alpha}, \alpha\right)=\mathcal{V}(x, \alpha)$. Therefore $E \in \mathcal{V}(x)\lceil D$ which was to be proved.

Let us now recall the definition of a $\mu$-dyadic system from [5].

Definition 4.10. If $X$ is a topological space a family $\{\langle A(\alpha, 0), A(\alpha, 1)\rangle$ : $\alpha \in \mu\}$ of pairs of closed subsets of $X$ is a $\mu$-dyadic system such that

1. $A(\alpha, 0) \cap A(\alpha, 1)=\emptyset$ for each $\alpha<\mu$,

2. for each $\epsilon \in \operatorname{Fn}(\mu, 2, \omega)$ we have $\bigcap_{\alpha \in \operatorname{dom}(\epsilon)} A(\alpha, \epsilon(\alpha)) \neq \emptyset$.

Theorem 4.11. If $D(c)$ holds, $X$ is a separable compact $T_{2}$ space of size $>c^{<c}$ then $X$ contains a c-dyadic system, consequently $X$ maps continuously onto $[0,1]^{c}$ ( and so $\beta \omega$ can be embedded into $X$ ).

Proof. Fix a countable dense subset $D$ of $X$. By lemma 4.9 there is a family $\mathcal{A}=\left\{A_{\alpha}, B_{\alpha}: \alpha<c\right\} \subset \mathcal{P}(D)$ such that $\overline{A_{\alpha}} \cap \overline{B_{\alpha}}=\emptyset$ for $\alpha<c$ and $\hat{\mathcal{A}}$ is centered. Let $D(\alpha, 0)=A_{\alpha}, D(\alpha, 1)=A_{\alpha}$ and $D(\alpha, n)=D$ for $\alpha<\kappa$ and $n \geq 2$ and consider the $\kappa \times \omega$-matrix $\mathcal{D}=\langle D(\alpha, i): \alpha<\kappa, i<\omega\rangle$. Since $\hat{\mathcal{A}}=\hat{\mathcal{D}}$ we can apply $D(c)$ to get a cofinal $S \subset c$ such that the family $\left\langle\overline{A_{\alpha}}, \overline{B_{\alpha}}: \alpha<c\right\rangle$ is $c$-dyadic. Now we can apply theorem [5, 3.18] to get the other consequences.

A topological space $X$ is called scattered if every subspace of $X$ has an isolated point. For a scattered space $X$ denote by $X^{(\alpha)}$ the $\alpha^{\text {th }}$ Cantor-Bendixon level of $X$. The height of $X, \operatorname{ht}(X)$, is defined as the minimal $\alpha$ with $X^{(\alpha)}=\emptyset$. Following [14] we call $X$ thin if all levels of $X$ are countable.

Since the cardinality of a locally compact, scattered separable space is at most $2^{\omega}$ by [13], the height of such a space is less then $\left(2^{\omega}\right)^{+}$. So under $\mathrm{CH}$ there is no such a space of height $\omega_{2}$. I. Juhász and $\mathrm{W}$. Weiss, 9, theorem 4], proved in ZFC that for every $\alpha<\omega_{2}$ there is a locally compact, scattered thin space $X$ with $h t(X)=\alpha$. M. Weese asked whether the existence of such a space of height $\omega_{2}$ follows from $\neg C H$. This question was answered in the negative by W. Just, who proved, [10, theorem 2.13 ], that if one adds Cohen reals to a model of $\mathrm{CH}$ then in the generic extension there are no locally compact scattered thin spaces of height $\omega_{2}$. On the other hand, J. Baumgartner and S. Shelah, [1], constructed a ZFC model which contains such a space of height $\omega_{2}$.

The next theorem is a generalization of the above mentioned result of Just.

Theorem 4.12. If $C^{s}(\kappa)$ holds then there is no locally compact, thin scattered space of height $\kappa$. 
Proof. Assume on the contrary that there is such a space $X$. We can assume that $X^{(\alpha)}=\{\alpha\} \times \omega$ for $\alpha<\operatorname{ht}(X)$. For each $\alpha<\operatorname{ht}(X)$ fix compact open neighbourhoods $U(\alpha, n)$ of $\langle\alpha, n\rangle$ for $n \in \omega$ such that $U(\alpha, n) \subset\{\langle\alpha, n\rangle\} \cup \bigcup\left\{X^{(\beta)}: \beta<\alpha\right\}$ and the sets $U(\alpha, n)$ for $n<\omega$ are pairwise disjoint.

Put $A(\alpha, 2 n)=U(\alpha, n) \cap X^{(0)}$ and $A(\alpha, 2 n+1)=X^{(0)} \backslash \bigcup\{U(\alpha, m)$ : $m \leq n\}$. Let

$$
T=\left\{t \in \omega^{<\omega}: t(0) \text { is even and } t(i) \text { is odd for } i>0\right\} .
$$

Now apply $C^{s}(\kappa)$ to the matrix $\langle A(\alpha, n): \alpha<\kappa, n<\omega\rangle \in \mathcal{M}(\kappa)$ and $T$.

Observe that $\quad A(\beta, 2 n) \cap \bigcap_{i<k} A\left(\alpha_{i}, 2 n_{i}+1\right)=\emptyset \quad$ iff

$$
\begin{gathered}
U(\beta, n) \cap X^{(0)} \subset \bigcup_{i<k} U\left(\alpha_{i}, n_{i}\right) \cap X^{(0)} \quad \text { iff } \\
U(\beta, n) \subset \bigcup_{i<k} U\left(\alpha_{i}, n_{i}\right) .
\end{gathered}
$$

Thus if $t=\left\langle 2 n, 2 n_{0}+1, \ldots, 2 n_{k-1}+1\right\rangle \in T$ and $\left\langle\beta, \alpha_{0}, \ldots, \alpha_{k-1}\right\rangle \in$ $(\kappa)^{k+1}$ then $A(\beta, 2 n) \cap \bigcap_{i<k} A\left(\alpha_{i}, 2 n_{i}+1\right)=\emptyset$ implies $\beta \leq \max _{i<k} \alpha_{i}$. This excludes 2.2(2). So 2.2(1) holds, that is we have a stationary set $S \subset \kappa$ such that if $t=\left\langle 2 n, 2 n_{0}+1, \ldots, 2 n_{k-1}+1\right\rangle \in T$ and $\left\langle\beta, \alpha_{0}, \ldots, \alpha_{k-1}\right\rangle \in(S)^{k+1}$ then

$$
A(\beta, n) \cap \bigcap_{i<k} A\left(\alpha_{i}, 2 n_{i}+1\right) \neq \emptyset,
$$

that is

$$
U(\beta, n) \backslash \bigcup_{i<k} \bigcup_{j \leq n_{i}} U\left(\alpha_{i}, j\right) \neq \emptyset .
$$

But $U(\beta, n)$ is compact and each $U(\alpha, n)$ is open so it follows that for every $\beta \in S$ and $n \in \omega$ the set

$$
D(\beta, n)=U(\beta, n) \backslash \bigcup\{U((\alpha, m): \alpha \in S \backslash\{\beta\} \wedge m \in \omega\}
$$

is not empty. For every such $\beta$ and $n$ let $\langle\gamma(\beta, n), m(\beta, n)\rangle \in D(\beta, n)$.

Since $X^{(\beta)}$ is dense in $X \backslash \bigcup\left\{X^{(\alpha)}: \alpha<\beta\right\}$ for every $\beta \in \kappa$ there is $k(\beta) \in \omega$ such that $\langle\beta, k(\beta)\rangle \in U\left(\beta^{*}, 0\right)$, where $\beta^{*}=\min S \backslash \beta+1$. Thus $\langle\beta, k(\beta)\rangle \notin D(\beta, k(\beta))$ and so $\gamma(\beta, k(\beta))<\beta$ for each $\beta \in S$. The set $S$ is stationary so there are a stationary set $S^{\prime} \subset S$, and ordinals $\gamma<\kappa$ and $k, m<\omega$ such that $k(\beta)=k, \gamma(\beta, k)=\gamma$ and $m(\beta, k)=m$ whenever $\beta \in S^{\prime}$. Thus $\langle\gamma, m\rangle \in D(\beta, k)$ for each $\beta \in S^{\prime}$, while $D(\beta, k) \cap D\left(\beta^{\prime}, k\right)=\emptyset$ for any $\left\{\beta, \beta^{\prime}\right\} \in\left[S^{\prime}\right]^{2}$ by the construction. This is a contradiction, hence the theorem is proved.

In [10] W. Just also proved that if one adds at least $\omega_{2}$ Cohen reals to a model of $\mathrm{CH}$ then in the generic extension there is no locally 
compact, scattered topological space $X$ such that $h t(X)=\omega_{1}+1$, $X^{(0)}$ is countable, $\left|X^{(\alpha)}\right| \leq \omega_{1}$ for $\alpha<\omega_{1}$ and $\left|X^{\left(\omega_{1}\right)}\right|=\omega_{2}$. The next theorem shows how to get a generalization of this result from our principles.

Theorem 4.13. If $\operatorname{cf}(\lambda) \geq \omega_{1}$ and $F\left(\lambda^{+}\right)$holds then there is no locally compact, scattered topological space $X$ such that $\operatorname{ht}(X)=\lambda+1, X^{(0)}$ is countable, $\left|X^{(\alpha)}\right| \leq \lambda$ for all $\alpha<\lambda$ and $\left|X^{(\lambda)}\right|=\lambda^{+}$.

Proof. Assume on the contrary that $X$ is such a space.

We can assume that $X^{(0)}=\omega$ and that $X^{(\lambda)}=\{\lambda\} \times \lambda^{+}$. For each $x \in X$ choose a compact open neighbourhood $U(x)$ of $X$ and let $B(x)=U(x) \cap \omega$. Put $\mathcal{B}=\left\{B(x): x \in X^{(\lambda)}\right\}$. Let $\mathcal{U}=\operatorname{CO}\left(X \backslash X^{(\lambda)}\right)$, i.e. the family of compact open subsets of $X \backslash X^{(\lambda)}=\bigcup\left\{X^{(\alpha)}: \alpha<\lambda\right\}$. and $\mathcal{F}=\{U \cap \omega: U \in \mathcal{U}\}$. Since $X$ is locally compact it follows that for each $\{x, y\} \in\left[X^{(\lambda)}\right]^{2}$ we have $U(x) \cap U(y) \in \mathcal{U}$ and so $B(x) \cap B(y) \in \mathcal{F}$.

Since $|\mathcal{F}| \leq \lambda$, it follows $\left|\mathcal{B}_{(2)}\right| \leq \lambda<\lambda^{+}$. Thus, applying theorem 4.7 for $k=2$ we can get a cofinal set $S \subset \lambda^{+}$such that the family

$$
\mathcal{I}=\left\{B(\langle\lambda, \alpha\rangle) \cap B(\langle\lambda, \beta\rangle):\{\alpha, \beta\} \in[S]^{2}\right\}
$$

is at most countable.

Then there is $\gamma<\lambda$ such that $\mathcal{I}$ is contained in $\operatorname{CO}\left(\cup\left\{X^{\left(\gamma^{\prime}\right)}: \gamma^{\prime}<\right.\right.$ $\gamma\})$. Therefore $U(y) \cap U\left(y^{\prime}\right) \subset \bigcup\left\{X^{\left(\gamma^{\prime}\right)}: \gamma^{\prime}<\gamma\right\}$ for each $\left\{y, y^{\prime}\right\} \in$ $[\{\lambda\} \times S]^{2}$ and so the sets $U(x) \cap X^{(\gamma)}$ for $x \in\{\lambda\} \times S$ are pairwise disjoint and non-empty which contradicts $\left|X^{(\gamma)}\right| \leq \lambda$.

Following the terminology of [4] a Hausdorff space is called $P_{2}$ if it does not contain two uncountable disjoint open sets. Hajnal and Juhász in [4 constructed a ZFC example of a first countable, $P_{2}$ space of size $\omega_{1}$ as well as consistent examples of size $2^{\omega}$ with $2^{\omega}$ as large as you wish. On the other hand, using a result of Z. Szentmiklóssy they proved that it is consistent with ZFC that $2^{\omega}$ is as large as you wish and there are no first countable $P_{2}$ spaces of size $\geq \omega_{3}$. However their method was unable to replace here $\omega_{3}$ with $\omega_{2}$. Our next result does just this because, as is shown in [4], every $P_{2}$ space is separable.

Theorem 4.14. If $C(\kappa)$ holds then every first countable, separable $T_{2}$ topological space $X$ of size $\kappa$ contains two disjoint open sets $U$ and $V$ of cardinality $\kappa$.

Proof. Let $D$ be a countable dense subset of $X$. For each $x \in X$ fix a neighborhood base $\{U(x, n): n \in \omega\}$ of $x$ in $X$. Apply $C(\kappa)$ to the matrix $\langle U(x, n) \cap D: x \in X, n<\omega\rangle$ and $T={ }^{2} \omega$. Since $X$ is $T_{2}$, 
there is no $S \in[X]^{\kappa}$ satisfying 2.2(1). So there are $S_{0}, S_{1} \in[X]^{\kappa}$ and $n, m \in \omega$ such that $U(x, n) \cap U(y, m) \cap D=\emptyset$ whenever $x \in S_{0}$ and $y \in S_{1}$. But $D$ is dense in $X$, therefore $U=\bigcup\left\{U(x, n): x \in S_{0}\right\}$ and $V=\bigcup\left\{U(y, m): y \in S_{1}\right\}$ are disjoint open sets of size $\kappa$.

Definition 4.15. Let $X$ be a topological space and $D \subset X$. We say that $D$ is sequentially dense in $X$ iff for each $x \in X$ there is a sequence $S_{x}$ from $D$ which converges to $x$. A space $Y$ is said to be sequentially separable if it contains a countable sequentially dense subset.

Definition 4.16. Given a topological space $\langle X, \tau\rangle$ and a subspace $Y \subset$ $X$ a function $f$ is called a neighbourhood assignment on $Y$ in $X$ iff $f: Y \longrightarrow \tau$ and $y \in f(y)$ for each $y \in Y$.

Our next result says that under $C(\kappa)$ if a sequentially separable space $X$ does not contain a discrete subspace of size $\kappa$, (i.e. $\hat{\mathrm{s}}(X) \leq \kappa$ using the notation of [5]) then $X$ does not contain left or right separated subspaces of size $\kappa$ either. This can be written as $\hat{\mathrm{h}}(X) \hat{\mathrm{z}}(X) \leq \kappa$. Since in [6] a normal, Frechet-Urysohn, separable (hence sequentially separable) space $X$ is forced such that $\mathrm{z}(X) \leq \omega_{1}$ but $\mathrm{h}(X)=\omega_{2}$, this result is not provable in $\mathrm{ZFC}$. First, however, we need a lemma.

Lemma 4.17. Assume that $C(\kappa)$ holds. Let $X$ be a sequentially separable space with $Y \subset X,|Y|=\kappa$. If $f$ is a neighbourhood assignment on $Y$ in $X$, then either (a) or (b) below holds:

(a) there is $Y^{\prime} \in[Y]^{\kappa}$ such that $f(y) \cap Y^{\prime}=\{y\}$ for each $y \in Y^{\prime}$ (hence $Y^{\prime}$ is discrete),

(b) there are $Y_{0}, Y_{1} \in[Y]^{\kappa}$ such that $y \in \overline{f(x)}$ whenever $x \in Y_{0}$ and $y \in Y_{1}$.

Proof. We can assume that $D=\omega$ is sequentially dense in $X$. For each $y \in Y$ choose a sequence $S_{y} \subset D$ converging to $y$. Let $A(y, 2 n)=$ $D \backslash f(y), A(y, 2 n+1)=S_{y} \backslash n, T=\{\langle 2 n, 2 m+1\rangle: n, m \in \omega\}$ and apply $C(\kappa)$. Assume first that $Y^{\prime} \in[Y]^{\kappa}$ witnesses 2.2(1) and let $x \neq y \in Y^{\prime}$. Then for each $n \in \omega$ we have $\left(S_{y} \backslash f(x)\right) \backslash n \neq \emptyset$, i.e. $S_{y} \backslash f(x)$ is infinite. But $S_{y}$ converges to $y$, so $y \notin f(x)$, and so $Y^{\prime}$ satisfies (a). Assume now that 2.2(2) holds. Then there are $Y_{0}, Y_{1} \in[\omega]^{\omega}$ and $m \in \omega$ such that $(D \backslash f(x)) \cap\left(S_{y} \backslash m\right)=\emptyset$ for each $x \in Y_{0}$ and $y \in Y_{1}$. But then $S_{y} \backslash m \subset f(x)$ hence $y \in \overline{f(x)}$ which was to be proved.

Theorem 4.18. If $C(\kappa)$ holds, $X$ is a regular, sequentially separable space with $\hat{\mathrm{s}}(X) \leq \kappa$ then $\hat{\mathrm{h}}(X) \hat{\mathrm{z}}(X) \leq \kappa$. 
Proof. Assume on the contrary that $Y \in[X]^{\kappa}$ and the neighbourhood assignment $f: Y \longrightarrow \tau_{X}$ witnesses that $Y$ is left (right) separated. We can assume that $Y=\kappa$ and $Y$ is left (right) separated under the natural ordering of $\kappa$. Since $X$ is regular we can find a neighbourhood assignment $g: Y \longrightarrow \tau$ with $\overline{g(y)} \subset f(y)$ for each $y \in Y$. Apply lemma 4.17 to $Y$ and $g$. Now 4.17(a) can not hold because $\hat{\mathrm{s}}(X) \leq \kappa$, hence there are $Y_{0}, Y_{1} \in[Y]^{\kappa}$ satisfying 4.17(b). Since both $Y_{0}$ and $Y_{1}$ are cofinal in $Y=\kappa$ under the natural ordering of the ordinals, applying left (or right) separatedness of $Y$ we can pick $x \in Y_{0}$ and $y \in Y_{1}$ such that $y \notin f(x)$. By the choice of $g$ this implies $y \notin \overline{g(x)}$ which contradicts 4.17(b).

The Sorgenfrey line $L$ is weakly separated and is of size $c$ with $\hat{\mathrm{s}}(L)=$ $\omega_{1}$. This shows that theorem 4.18 does not remain valid if you put weakly separated subspaces instead of right or left separated ones.

As an easy consequence of 4.18 we get the following result in which (sequential) separability is no longer assumed. We also note that under $\mathrm{CH}$ the assumption of $X$ being Frechet-Urysohn is not necessary in this result.

Theorem 4.19. Assume $C\left(\omega_{2}\right)$. If $X$ is regular, Frechet-Urysohn space and $\mathrm{s}(X)=\omega$ then $\mathrm{h}(X) \leq \omega_{1}$.

Proof. If $C\left(\omega_{2}\right)$ and $X$ is separable, then by theorem 4.18 even $\mathrm{s}(X) \leq$ $\omega_{1}$ implies $\mathrm{h}(X) \mathrm{z}(X) \leq \omega_{1}$. Now, every uncountable space $X$ which is both right and left separated contains an uncountable discrete subspace, hence every right separated subspace of $X$ is (hereditarily) separable. So by the above if $Y \subset X$ is right separated then $|Y| \leq \omega_{1}$, i.e. $\mathrm{h}(X) \leq \omega_{1}$.

In [8] we investigated the following question: What makes a space have weight larger than its character? To answer this question we introduced the notion of an irreducible base of a space and proved that any weakly separated space has such a base, moreover the weight of a space possessing an irreducible base can not be smaller than its cardinality. We asked [8, Problem 1] whether every first countable space of uncountable weight contains an uncountable subspace with an irreducible base? In theorem 4.23 and corollary 4.24 we will give a partial positive answer to this problem, using the principle $C(\kappa)$. First we recall some definitions from [8].

Definition 4.20. Let $X$ be a topological space. A base $\mathcal{U}$ of $X$ is called irreducible if it has an irreducible decomposition $\mathcal{U}=\bigcup\left\{\mathcal{U}_{x}: x \in X\right\}$, i.e., $(i)$ and $(i i)$ below hold: 
(i) $\mathcal{U}_{x}$ is a neighbourhood base of $x$ in $X$ for each $x \in X$,

(ii) for each $x \in X$ the family $\mathcal{U}_{x}^{-}=\bigcup_{y \neq x} \mathcal{U}_{y}$ is not a base of $X$ (hence $\mathcal{U}_{x}^{-}$does not contain a neighbourhood base of $x$ in $\left.X\right)$.

Definition 4.21. Let $X$ be a topological space with $Y \subset X$. Similarly as above, an outer base $\mathcal{U}$ of $Y$ in $X$ is called irreducible if it has an irreducible decomposition $\mathcal{U}=\bigcup\left\{\mathcal{U}_{y}: y \in Y\right\}$, i.e., (i) and (ii) below hold:

(i) $\mathcal{U}_{y}$ is a neighbourhood base of $y$ in $X$ for each $y \in Y$,

(ii) for each $y \in Y$ the family $\mathcal{U}_{y}^{-}=\bigcup\left\{\mathcal{U}_{z}: z \in Y \backslash\{y\}\right\}$ does not contain a neighbourhood base of $y$ in $X$.

Note that in general, a subspace $Y$ having an irreducible outer base in $X$ does not necessarily possess an irreducible base in itself. However, if $Y$ is dense in an open set and the irreducible outer base of $Y$ consists of regular open sets then clearly this is the case. Moreover, by our next result, under certain conditions we can at least find another subspace of the same size as $Y$ that does have an irreducible base.

Lemma 4.22. If $X$ is a regular, separable space and $Y \subset X$ has an irreducible outer base in $X$ consisting of regular open sets, then there is $Z \subset X$ with $|Z|=|Y|$ such that the subspace $Z$ has an irreducible base.

Proof. Let $\mathcal{B}=\bigcup\left\{\mathcal{B}_{y}: y \in Y\right\}$ be an irreducible outer base of $Y$ in $X$ consisting of regular open sets and $D$ be a countable dense subset of $X$. We distinguish two cases:

Case 1. $|(\operatorname{int} \bar{Y}) \cap Y|=|Y|$.

Let $Z=(\operatorname{int} \bar{Y}) \cap Y$. Since $Z$ is dense in the open set int $\bar{Y}$, by our above remark $Z$ has an irreducible base.

Case 2. $|(\operatorname{int} \bar{Y}) \cap Y|<|Y|$.

In this case the set $Y_{1}=Y \backslash \operatorname{int} \bar{Y}$ is nowhere dense, so $D_{1}=D \backslash \overline{Y_{1}}$ is dense in $X$. Let $Z=D_{1} \cup Y_{1}$, then $|Z|=\left|Y_{1}\right|=|Y|$. Write $D_{1}=\left\{d_{n}: n<\omega\right\}$ and for each $d_{n} \in D$ let $\mathcal{B}_{d_{n}}$ be a neighbourhood base of $d_{n}$ in $X$ consisting of regular open sets, that are disjoint to $Y_{1} \cup\left\{d_{m}: m<n\right\}$. Then clearly $\cup\left\{\mathcal{B}_{z}: z \in Z\right\}$ is an irreducible outer base of $Z$ in $X$ consisting of regular open sets and $Z$ is dense in $X$, so again we are done.

Theorem 4.23. Let $\kappa$ be a regular cardinal and assume $C(\kappa)$. If $X$ is a separable, first countable, regular space with $\mathrm{w}(X) \geq \kappa$, then there is subspace $Y \subset X$ of cardinality $\kappa$ that has an irreducible base. 
Proof. Let $D \subset X$ be a countable, dense subset of $X$. For each $x \in X$ fix a neighbourhood base $\{U(x, n): n \in \omega\}$ consisting of regular open sets and set $V(x, n)=U(x, n) \cap D$. Since the $U(x, n)$ are regular open and $D$ is dense, we clearly have $U(x, n) \subset U(y, m)$ iff $V(x, n) \subset$ $V(y, m)$.

Since $\mathrm{w}(X) \geq \kappa$, by transfinite recursion on $\beta<\kappa$ we can choose points $\left\{x_{\alpha}: \alpha<\kappa\right\} \subset X$ such that for any $\beta<\kappa$ the family $\left\{U\left(x_{\alpha}, n\right)\right.$ : $\alpha<\beta, n<\omega\}$ does not contain a neighbourhood base of $x_{\beta}$, in other words, there is a natural number $k_{\beta}$ such that for all $\alpha<\beta<\kappa$ and $n \in \omega$ we have

$$
\neg\left(x_{\beta} \in U\left(x_{\alpha}, n\right) \subset U\left(x_{\beta}, k_{\beta}\right)\right) .
$$

We can assume that $k_{\beta}=0$ for each $\beta<\kappa$. Let $X^{\prime}=\left\{x_{\alpha}: \alpha<\kappa\right\}$. For $x \in X^{\prime}$ and $n<\omega$ put

$$
A(x, 2 n)=[V(x, n) \times\{0\}] \cup[(D \backslash V(x, n)) \times\{1\}]
$$

and

$$
A(x, 2 m+1)=[(D \backslash V(x, 0)) \times\{0\}] \cup[V(x, m) \times\{1\}] .
$$

Note that $A(x, 2 n) \cap A(y, 2 m+1)=\emptyset$ iff $V(y, m) \subset V(x, n) \subset V(y, 0)$. Apply $C(\kappa)$ to $\left\langle A(x, i): x \in X^{\prime}, i<\omega\right\rangle$ and $T=\{\langle 2 n, 2 m+1\rangle: n, m<$ $\omega\}$. By $(*)$ (and $k_{\beta}=0$ ) there are no $D, E \in\left[X^{\prime}\right]^{\kappa}$ and $n, m \in \omega$ such that

$$
V(y, m) \subset V(x, n) \subset V(y, 0)
$$

whenever $x \in D$ and $y \in E$, because $(\dagger)$ fails if $x=x_{\alpha}, y=x_{\beta}$ and $\alpha<\beta$. So there is $Y \in\left[X^{\prime}\right]^{\kappa}$ such that for all $n, m \in \omega$ and $x \neq y \in Y$ the intersection of $A(x, 2 n)$ and $A(y, 2 m+1)$ is not empty. This means that $\neg(V(y, m) \subset V(x, n) \subset V(y, 0))$, i.e. if we set $\mathcal{B}_{y}=\{U(y, n): n<$ $\omega\}$ then it follows that $\mathcal{B}=\bigcup\left\{\mathcal{B}_{y}: y \in Y^{\prime}\right\}$ is a an irreducible outer base of $Y$ in $X$ consisting of regular open sets. Now applying lemma 4.22 we can conclude the proof.

Unfortunately, as $C\left(\omega_{1}\right)$ is false, the above result is not applicable in the perhaps most interesting case when $\mathrm{w}(X)=\omega_{1}$. The annoying assumption of separability, however, can be circumvented as follows.

Corollary 4.24. Assume $C(\kappa)$. If $X$ is a first countable, regular space with $\mathrm{w}(X) \geq \kappa$, then there is an uncountable subspace $Y \subset X$ that has an irreducible base.

Proof. If $X$ is separable, then the previous theorem can be applied. If $X$ is not separable, then $X$ contains an uncountable left separated subspace $Y$ and again $Y$ has an irreducible base. 


\section{REFERENCES}

[1] J. E. Baumgartner, S. Shelah, Remarks on superatomic Boolean algebras, Ann. Pure Appl. Logic, 33 (1987), no. 2, 119-129.

[2] A. Dow, Large compact separable spaces may all contain $\beta N$, Proc. Amer. Math. Soc. 109 (1990) no.1. 275-279.

[3] A. Dow , On initially $\kappa$-compact spaces, in: Rings of continuous functions, ed. C.E. Aull, Lecture Notes in pure and applied Mathematics, 1985., v.95, Marcel Dekker, Inc, New York and Basel, pp. 103-108.

[4] A. Hajnal, I. Juhász, Intersection properties of open sets, Topology and its Application, 19 (1985), 201-209.

[5] I. Juhász Cardinal functions in topology - ten years later, Mathematical Centre Tracts 123, Amsterdam, 1980.

[6] I. Juhász, L. Soukup, How to force a countably tight, initially $\omega_{1}$ compact but non-compact space? to appear Topology and its Applications

[7] I. Juhász, Zs. Nagy, L. Soukup, Z. Szentmiklóssy, Intersection properties of open sets, II. to appear in the Proceedings of the Tenth Summer Conference in General Topology and Applications, TU Delft, Netherlands.

[8] I. Juhász, L. Soukup, Z. Szentmiklóssy, What makes a space have large weight? "Topology AND its Applications" 57 (1994), no 2-3, 271-285.

[9] I. Juhász, W. Weiss, On thin-tall scattered spaces, Colloquium MatheMATICUM, vol XL (1978) pp 63-68.

[10] W. Just, Two consistency results concerning thin-tall Boolean algebras Algebra Universalis 20 (1985) no.2, 135-142.

[11] K. Kunen, Set Theory, North-Holland, New York, 1980.

[12] K. Kunen, Inaccessibility Properties of Cardinals, Doctoral Dissertation, Stanford, 1968.

[13] S. Mrówka, M. Rajagopalan, T. Soudararajan, A characterization of compact scattered spaces through chain limits (chain compact spaces), Proceedings of TOPO 72, Pittsburg 1972, Lecture Notes, berlin 1974.

[14] Judy Roitman, Height and weight of superatomic Boolean algebras, Proc. Amer. Math. Soc. 94 (1985), no 1, 9-14.

[15] W. Weiss, Versions of Martin's Axiom, in Handbook of Set-Theoretic Topology, ed. K. Kunen and J.E. Vaughan, Elseview Science Publisher, B.V. 1984.

Mathematical Institute of the Hungarian Academy of Sciences

E-mail address: juhasz@math-inst.hu

Mathematical Institute of the Hungarian Academy of Sciences

E-mail address: soukup@math-inst.hu

EÖtvös Loránd University, Department of Analysis

E-mail address: szetmiklossy@math-inst.hu 